\title{
Transcanalicular endoscopic primary dacryoplasty for congenital nasolacrimal duct obstruction
}

\author{
Nozomi Matsumura $\mathbb{1}^{1} \cdot$ Toru Suzuki $^{2} \cdot$ Satoshi Goto ${ }^{3} \cdot$ Takeshi Fujita $^{1} \cdot$ Shin Yamane ${ }^{4} \cdot$ Maiko Maruyama-Inoue $^{4}$. \\ Kazuaki Kadonosono ${ }^{4}$
}

Received: 15 September 2018 / Revised: 20 January 2019 / Accepted: 31 January 2019 / Published online: 19 February 2019

(c) The Royal College of Ophthalmologists 2019

\begin{abstract}
Objectives To examine the causes of congenital nasolacrimal duct obstruction (CNLDO) using dacryoendoscopy, and to evaluate the surgical outcomes of primary transcanalicular endoscopic dacryoplasty.

Methods The subjects of this study were a total of 56 eyes of 46 Japanese children aged one to five years old (mean, $29.1 \pm$ 14.0 months old) with clinically diagnosed CNLDO. The blockage was visualized and probed using a dacryoendoscope (MD10 with a $20 \mathrm{G}$ probe, Fiber Tech Co., Ltd., Japan) under general anesthesia. We used a self-retaining bicanalicular lacrimal stent (Lacrifast ${ }^{\circledR}$, Kaneka Co., Ltd., Japan) for nasolacrimal duct intubation.

Results In each case the obstruction was found to be caused by a single focal blockage at the distal end of the duct. A nasolacrimal dacryolith was observed in 5 eyes $(9 \%)$ and successfully removed using the dacryoendoscope. The success rate of probing by subsequent nasolacrimal duct intubation was $100 \%$. No complications were observed.

Conclusions We obtained a $100 \%$ success rate with primary transcanalicular endoscopic dacryoplasty for the treatment of CNLDO. Direct visualization inside the lacrimal passage allowed for precise probing, even in infants, leading to successful treatment of CNLDO without any complications.
\end{abstract}

\section{Introduction}

Congenital nasolacrimal duct obstruction (CNLDO) is considered to be the most common cause of epiphora in the paediatric population [1-5]. It is histopathologically characterized by a membranous blockage around the distal end of the nasolacrimal duct $[6,7]$. The most common treatment for CNLDO is probing, although the timing is controversial, due to high frequency of the spontaneous resolution of symptom during the first year of life [1-5, 8]. Recently, the dacryoendoscope has become available for lacrimal passage

Nozomi Matsumura

kcmceyecare@gmail.com

1 Department of Ophthalmology, Kanagawa Children's Medical Center, Yokohama City, Japan

2 Suzuki Eye Clinic, Kitakyushu City, Japan

3 Department of Ophthalmology, The Jikei University School of Medicine, Tokyo, Japan

4 Department of Ophthalmology and Micro-technology, Yokohama City University Medical Center, Yokohama City, Japan examination and treatment in adults [9-18]. However, to the best of our knowledge, there are few reports about probing using a dacryoendoscope for CNLDO [9, 11-13]. As demonstrated in the 3 cases of paediatric lacrimal duct obstruction reported by us [14], dacryoendoscopy allows for direct visualization of the lacrimal passage even in infants. Herein, we report the endoscopic findings of CNLDO and the surgical outcomes of primary transcanalicular endoscopic dacryoplasty.

\section{Subjects and Methods}

\section{Patients and assessments}

This study was a prospective, non-comparative, singlecenter, consecutive case study. All of the patients were treated at Kanagawa Children's Medical Center, Yokohama City, between January 2011 and October 2017. We performed primary transcanalicular endoscopic dacryoplasty a total of 56 eyes of 46 consecutive Japanese children aged from one to five years old (mean, 29.1 \pm 14.0 months old) with clinically diagnosed CNLDO. We obtained approval 


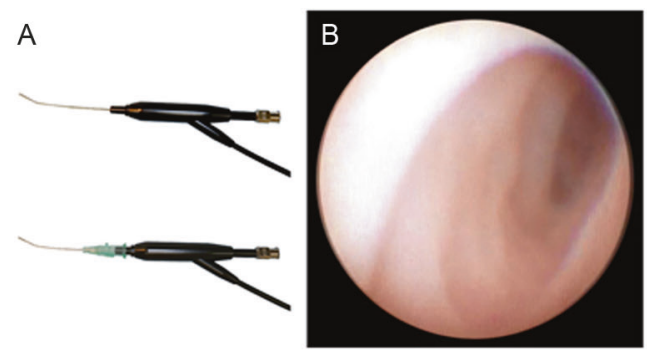

Fig. 1 From: Dacryoendoscopy and normal findings for a paediatric nasolacrimal duct. a A Dacryoendoscopic probe (top). We used an 18$\mathrm{G}$ intravenous catheter (sheath) fitted onto the probe (bottom). b Lumen of the nasolacrimal duct. A pinkish mucosa with multiple
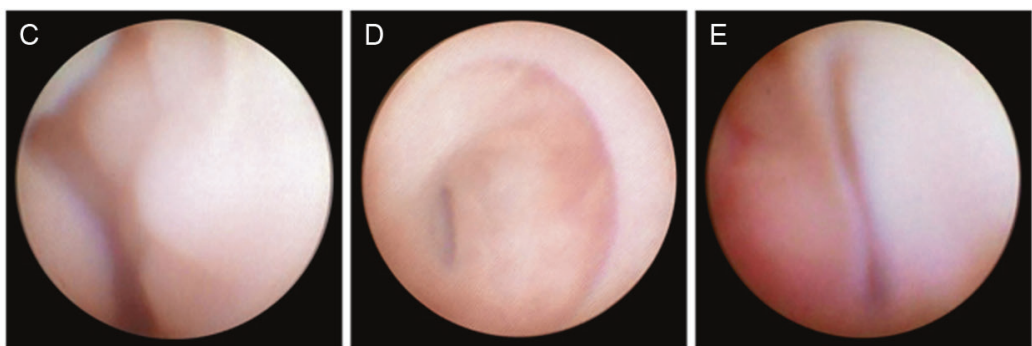

spiral-like folds is visible. c Irregular bumpy structures like follicles are often seen in normal paediatric nasolacrimal ducts. d The physiological ostium is located inside (nasal) the distal end of the duct (Right eye). e A close-up image of the ostium for this study from our Institutional Review Board, and all of investigations adhered to the tenets of the Declaration of Helsinki. Informed consent was obtained from the guardian of each child.

We excluded children with any previous history of surgical intervention, any trigger for onset the obstruction, such as viral infection, as represented by epidemic keratoconjunctivitis (EKC), any punctal or canalicular obstructions, and any systemic syndromes and/or facial anomalies.

A clinical diagnosis of lacrimal duct obstruction was made when we observed at least one of the characteristic symptoms, including epiphora and sticky eye due to mucous discharge, with a positive result of the fluorescein dye retention test (FDRT). Treatment success was defined as disappearance of the symptom and a negative FDRT, which was evaluated by an ophthalmologist blinded to the background characteristics of the patients. The surgical outcome was evaluated six months after the stent removal.

\section{Instrument}

We used a dacryoendoscope, which is a microendoscope specially designed for examination and treatment of the lacrimal passages (RUIDO fiberscope MD10; FiberTech Co., Ltd., Tokyo, Japan) (Fig. 1 top left). The dacryoendoscope has a curved rigid probe of 0.9 -mm diameter (20 G), containing a 10,000 pixel fiberoptic bundle, illumination fibers, and an irrigation channel. After dilating the punctum, we inserted the dacryoendoscope probe into the duct through the canalicular system. In order to obtain good visualization while performing the endoscopy, we expanded the lacrimal outflow system by saline irrigation through the endoscope channel. Also, in order to probe the blockage as the treatment, we used an $18 \mathrm{G}$ intravenous indwelling catheter (sheath) fitted onto the endoscope probe (Fig. 1a).

\section{Surgery}

We performed the procedure under general anesthesia in all the cases. At first, we identified the sac lumen before examining the nasolacrimal duct. Then, we introduced the dacryoendoscope into the nasolacrimal duct and proceeded to confirm the cause of the CNLDO. We used the dacryoendoscope sheath fitted onto the endoscopic probe for probing the blockage. Finally we performed nasolacrimal duct intubation using a self-retaining bicanalicuar lacrimal stent (Lacrifast $^{\circledR}$ : Japan, Lacriflow ${ }^{\circledR}$ : U.S.A., 90 mm type, Kaneka Co., Ltd., Osaka, Japan) [19]. After placing the lacrimal stent, we inserted the endoscope alongside the stent in the duct and confirmed the stent positioning to exclude false passage intubation. We left the stent in situ for 4 weeks, and removed it through the punctum in an office setting.

\section{Results}

\section{Examination findings}

On dacryoendoscopy, paediatric nasolacrimal ducts typically had a pinkish mucosa with multiple spiral folds (Fig. 1b) or irregular bumpy structures (Fig. 1c). The ostium was located on the medial wall around the end of the duct (Fig. 1d). Usually the ostium could be seen as a gap, like a slit, in the antero-posterior direction, in the duct lumen (Fig. 1e).

In all of the cases of CNLDO included in the study, the obstruction was caused by a single focal blockage. We could probe the blockage using the sheath while performing the endoscopy, which yielded much information about the characteristics of the CNLDO. According to the endoscopic findings, we divided the CNLDO into 2 categories: the simple and complicated types (Table 1).

The simple type, accounted for $73 \%$ of all the cases, and was characterized by a thin blockage. We could divide this type of blockage into two subtypes, including the membrane and cleft subtypes. Under the high hydraulic pressure of syringing, a slightly concave-shaped blockage was seen in the membrane subtype (61\%) (Figs. 2a, b, and 3a), while a cleft-shaped blockage was seen in the cleft subtype (13\%) 
(Fig. 3c). In both the subtypes, the nasal cavity could easily be reached without demonstrable hemorrhage. Following

Table 1 Classification of congenital nasolacrimal duct obstruction by dacryoendoscopic findings

\begin{tabular}{lcl}
\hline Type $(n=56)$ & Number of eyes $(\%)$ & Mean months of age \\
\hline Simple & $41(73 \%)$ & $29 \pm 19$ \\
Membrane & $34(61 \%)$ & $29 \pm 15$ \\
Cleft & $7(13 \%)$ & $30 \pm 19$ \\
Complicated & $15(27 \%)$ & $29 \pm 7$ \\
Stenosis & $8(14 \%)$ & $31 \pm 8$ \\
Fibrosis & $7(13 \%)$ & $26 \pm 6$ \\
Dacryolith & $5(9 \%)$ & $23 \pm 8$ \\
\hline
\end{tabular}
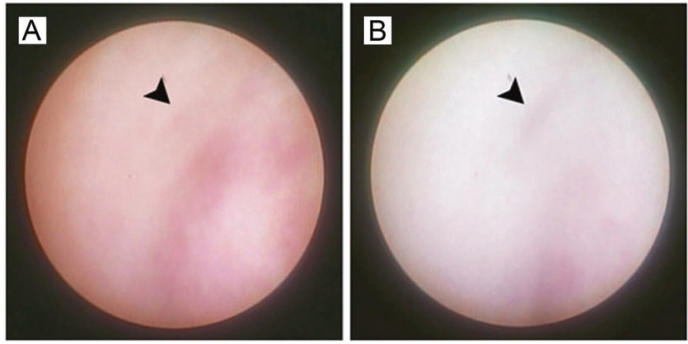

Fig. 2 From: Continuous images obtained during dacryoendoscopic probing for CNLDO. a Image of a typical case of membranous obstruction. The opening of the nasolacrimal duct is covered by mucous membrane (arrowhead). b Image obtained during highpressure irrigation. Irrigation under a strong hydraulic pressure revealed the precise location, which was slightly concave, to be probed
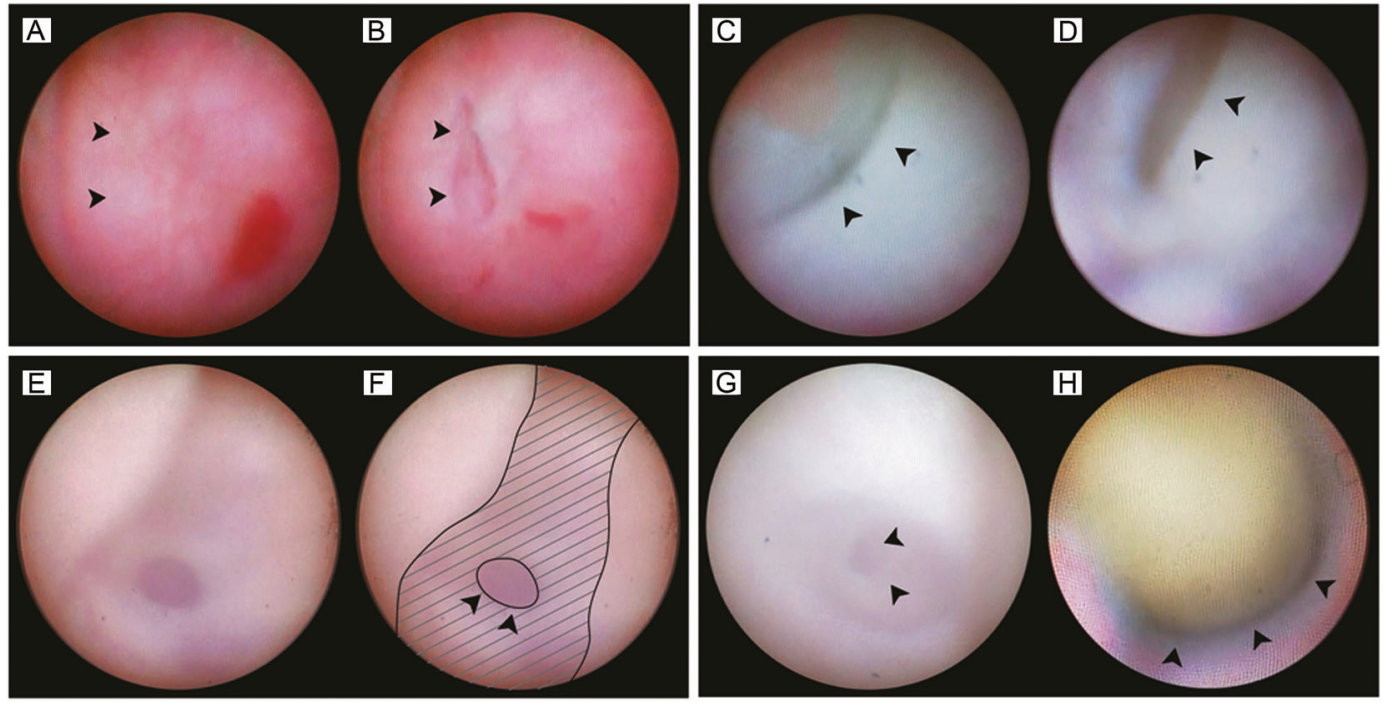

Fig. 3 From: Typical dacryoendoscopic images of CNLDO. a A typical membrane-type CNLDO before perforation (Right Eye). The surface is smooth and capillaries are observed continuously (arrowhead). b Image after probing for (a). The ostium has emerged (arrowhead). c Image of a cleft-type CNLDO before probing (arrowhead). d After probing for (c). The ostium has become visible (arrowhead). e Close-up image of an example of a stenosis subtype of

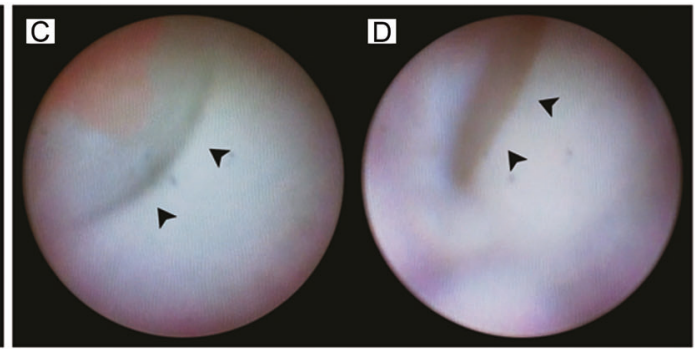

the probing, we could see a slit-shaped ostium, as well as in physiological cases (Figs. 2c, d, 3b, d).

The blockage in the complicated type, which accounted for $27 \%$ of the cases, was different in nature from that in the simple type. This type could also be divided into two subtypes, namely, the stenosis and fibrosis subtypes. In the stenosis subtype, syringing revealed partial patency, and any of various endoscopic findings, as follows. (1) incomplete opening of the physiological ostium was observed, including a pinhole-shaped opening, which was smaller in diameter than the diameter of the dacryoendoscope $(0.9 \mathrm{~mm})($ Fig. 3e, f); (2) the residue of a dacryolith with or without a stenotic ostium (Fig. 3h); (3) extremely narrow inferior nasal meatus, mainly caused by allergic rhinitis with
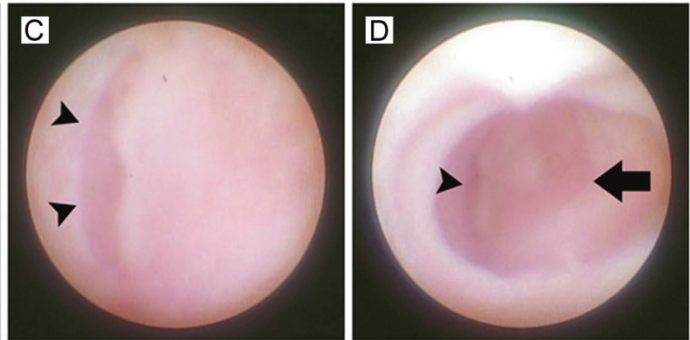

(arrowhead). c A close-up image obtained just after probing. An ostium in the sagittal direction has become visible. d A distant image after perforation. The ostium is located inside (nasal) the nasolacrimal duct, slightly proximal to the distal end (arrowhead). A "pocket" at the end of the duct is seen (arrow)

CNLDO. f Image description of (e). A small pinhole-shaped stenotic hole (arrowhead) is observed in a part of the obstructed physiological ostium (slanted line). $\mathrm{g}$ Image of the duct in a case of fibrosis-type CNLDO; a "dimple," indicating the thinner part of the blockage is visible (arrowhead). h A yellow dacryolith (arrowhead) is visible in the lacrimal duct lumen 

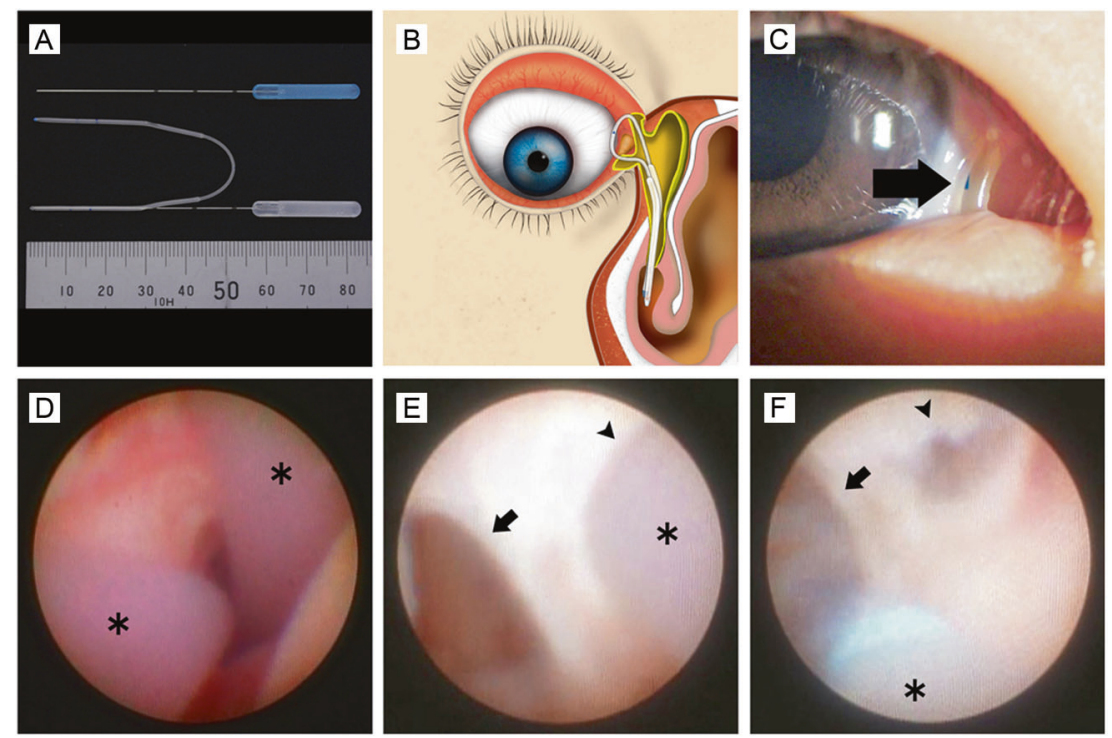

Fig. 4 From: Nasolacrimal duct intubation assisted by dacryoendoscopy. a A self-retaining bicanalicular lacrimal stent (Lacrifast ${ }^{\circledR}$, short type, Kaneka Co., Ltd., Osaka, Japan). b Illustration of the selfretaining bicanalicular lacrimal stent positioned in the duct. c A slitlump examination after intubation and insertion of the lacrimal stent in an infant. d Image of a successfully inserted bicanalicular stent (*).

edematous mucosa, or chronic paranasal sinusitis with thickened nasal mucosa, with or without stenosis of the ostium.

In the fibrosis subtype, a focal fibrous blockage was observed at the end of the duct (Fig. 3g). Similar to the case in the simple-type, the blockage could be probed but it was harder than simple-type obstruction.

We identified a dacryolith in 5 cases (9\%) (Fig. 3h). In each of these cases, we performed dacryolith fragmentation and successfully removed the fragments through the duct using the sheath of the scope. One of the five cases showed no stenosis at the distal end of the duct despite syringing showing partial patency. This was thought to be a case of a residual dacryolith after spontaneous resolution of the CNLDO.

There were no cases in the present study that could be classified into the conventional complex CNLDO group which was reported in past literature, including generalized blockage of the duct, sac fibrosis, nasolacrimal canal dysplasia or aplasia, buried probes, or bony obstruction.

Following visualization of the blockage, we could probe the blockage in all the cases and remove the dacryolith. At the same time, we performed nasolacrimal duct intubation in all the cases. After intubation, the precise stent positioning was confirmed by dacryoendoscopy from the side of the already inserted tube (Fig. $4 \mathrm{a}-\mathrm{d}$ ). The stent positioning could be corrected if needed (Fig. 4e, f). As a result, false passage intubation was eliminated in the present study. e An image of unsuccessful intubation. The stent is stuck in the mucosa (arrowhead). The correct way is shown on the left side (arrow). f Correction of unsuccessful intubation. We pulled out the stubbed stent, and guided it in the correct manner using a dacryoendoscope. The false passage created by the stent is visible (arrowhead)

\section{Surgical outcomes}

The success rate of transcanalicular primary endoscopic dacryoplasty at 6 months after the treatment was $100 \%$. No complications were observed in this case series.

\section{Discussion}

Dacryoendoscopy allowed direct visualization and diagnosis of the cause of the lacrimal duct obstruction, even in infants. In every case, the cause was a single and focal blockage around the end of the duct, which is consistent with the conclusions of previous histopathological studies $[6,7]$. The precise location of the blockage in the simple type was sometimes just about 0.5 to $1.0 \mathrm{~mm}$ proximal to the end of the duct. This finding is unique to our study. From our experience of dacryoendoscopy in adult patients, the physiological duct usually ends with a "pocket" in the nasal mucosa of the lateral wall of the nasal cavity, with the ostium adjacent to the pocket proximally. We have confirmed that the physiological structure of the end of the duct was even in cases of CNLDO. This particular anatomical structure is considered to be a factor contributing to failedprobing using the blind technique. A metal probe is considered to be easily misdirected to the pocket instead of striking the ostium. By using dacryoendoscopy, we could avoid misdirected probing, leading to a high success rate. 
Mac and colleagues conducted a CNLDO study using a nasal endoscope [20]. They reported that $65 \%$ cases of CNLDO in their case series showed a membranous obstruction at the lower site of the duct. We found a membranous blockage in $61 \%$ of our cases of CNLDO using the dacryoendoscope, which is compatible with the conclusion of the aforementioned study. In addition, we found similar blockage to the membranous-type, which was named the cleft-type blockage, accounting for $13 \%$ of a total. Since the cleft-type blockages were located at the same site as the membranous-type blockages, we categorized the cleft and membranous type of blockages into the same group (simple type). Both the membranous blockage in Mac's study and simple-type blockage in our study are considered to be amenable to successful probing treatment, as long as misguided probing is avoided.

In regard to the frequency of the complex type of CNLDO, which is diagnosed by conventional probing, it is said to occur at an incidence of 2.2-3.6\% in children under 24 months of age and of $20-57 \%$ in children between 24 and 64 months of age; this type of CNLDO is considered as the main cause of failed probing [21]. However, there was no case of the conventional complex type of CNLDO in this study, despite the average age of the children included in the study of 29 months. There is a possibility that many of the cases of conventional complex CNLDO result from misguided probing, which could be avoided by using a dacryoendoscope.

The stenosis subtype of CNLDO, in other words, incomplete obstruction, was previously detected among cases of infantile epiphora by nasal endoscopy [8, 22-24] or computed tomography (CT) [25]. The prevalence of this type of blockage was $14 \%$ in our study and $15 \%$ in Mac's study [20]. We could clarify three different groups of the stenosis subtype of clinical CNLDO in our study, namely, incomplete opening, residual dacryolith, and extremely narrow inferior nasal meatus accompanied by rhinitis. In regard to treatment, probing and intubation were suitable for incomplete openings, removing of the dacryolith was appropriate for residual dacryoliths, and intubation followed by medical treatment for rhinitis was effective for extremely narrow inferior nasal meatus. We were able to select the optimal treatment based on the findings in the stenosis type, which was an advantage of using dacryoendoscopy.

In regard to the fibrosis type of CNLDO, we previously reported that the tear-fluid levels of interleukin (IL)-6 were significantly higher on the involved side as compared to the control side, in paediatric cases of lacrimal duct obstruction [26]. Because IL-6 has been reported to be involved in the conversion of acute inflammation to a chronic profibrotic state [27], sustained elevation of the concentrations of IL-6 in the tear fluid might drive the development of severe inflammatory fibrosis in cases of CNLDO.
We found a dacryolith in 5 eyes $(9 \%)$ in the present study. CNLDO have rarely reported to be complicated with dacryoliths $[9,13]$. We could successfully remove the dacryolith in all of the 5 cases while performing endoscopic probing.

PEDIG's prospective study showed that the success rate of simple probing without using an endoscope for CNLDO aged from 12 to 15 months was $82 \%$ [28]. On the other hand, our prospective study to evaluate the success rate of primary endoscopic dacryoplasty for CNLDO aged from 1 to 5 years old showed that the treatment outcome was $100 \%$. Although there wasn't age-matched in 2 reports, we consider that our treatment was more effective than a conventional simple probing.

Dacryoendoscopy is useful for understanding the differences in the etiologies of obstruction, leading to selection of the most appropriate treatment based on the endoscopic findings. Treatment procedures such as simple probing, intubation, balloon dilatation, topical or systemic medications, and dacryocystorhinostomy (DCR) may be used alone or in combination according to the type of obstruction. All of the cases of the simple-type obstruction may not require intubation after probing. The complicated type may require intubation. Although a further comparative study is required, we could find etiological diversity in the CNLDO and potential treatment decision using dacryoendoscopy.

We consider that there may be the following four advantages of using the dacryoendoscope. Firstly, we could visualize the blockage exactly and categorize them. Secondly, we could probe them with avoiding false passage creation, leading the better treatment outcome than that of the conventional method. Thirdly, dacryoliths could be detected and removed. Finally, the optimal treatment could be selected based on the dacryoendoscopic findings.

There were some limitations of the present study. We were unable to strictly confirm the "congenital" nature of the nasolacrimal duct obstruction in this study. We excluded complex cases of paediatric epiphora, such as cases of failed probing, acquired lacrimal duct obstruction, canalicular obstruction, and systemic syndromes and/or facial anomalies, from the present study. Thus, further accumulation of cases is necessary.

In conclusion, we obtained a $100 \%$ success rate of transcanalicular endoscopic dacryoplasty for CNLDO. Direct visualization inside the lacrimal passage allowed for precise probing and intubation, even in infants, leading to successful treatment of CNLDO without any complications.

\section{Study Highlights}

\section{What was known before}

- The success rates of probing for congenital nasolacrimal duct obstruction by blind technique were reported about $70-90 \%$. The cause of failure had not been clear. 


\section{What this study adds}

- Direct visualization inside the lacrimal passage by dacryoendoscopy allowed for precise probing. We obtained a $100 \%$ success rate with primary transcanalicular endoscopic dacryoplasty for the treatment of congenital nasolacrimal duct obstruction.

\section{Compliance with ethical standards}

Conflict of interest TS has a patent on dacryoendoscopy with Fiber Tech Co., Ltd., Japan. The remaining authors declare that they have no conflict of interest.

Publisher's note: Springer Nature remains neutral with regard to jurisdictional claims in published maps and institutional affiliations.

\section{References}

1. Young JD, MacEwen CJ. Managing congenital lacrimal obstruction in general practice. BMJ. 1997;315:293-6.

2. Kapadia MK, Freitag SK, Woog JJ. Evaluation and management of congenital nasolacrimal duct obstruction. Otolaryngol Clin North Am. 2006;39:959-77.

3. MacEwen CJ, Young JD. Epiphora during the first year of life. Eye. 1991;5:596-600.

4. MacEwen CJ. Congenital nasolacrimal duct obstruction. Compr Ophthalmol Update. 2006;7:79-87.

5. Takahashi Y, Kakizaki H, Chan WO, Selva D. Management of congenital nasolacrimal duct obstruction. Acta Ophthalmol. 2010;88:506-13.

6. Sevel D. Development and Congenital Abnormalities of the Nasolacrimal Apparatus. J Pediatr Ophthalmol Starbismus. 1981;18:13-9.

7. Cassaday JV. Developmental anatomy of nasolacrimal duct. AMA Arch Ophthalmol. 1952;47:141-58.

8. Cibis GW. Nasolacrimal duct probing in infants. Ophthalmology. 1979;86:1488-91.

9. Sasaki H, Takano T, Murakami A. Direct endoscopic probing for congenital lacrimal duct obstruction. Clin Exp Ophthalmol. 2013;41:729-34.

10. Mullner K, Bodner E, Mannor GE. Endoscopy of the lacrimal system. Br J Ophthalmol. 1999;83:949-52.

11. Kato K, Matsunaga K, Takashima Y, Kondo M. Probing of congenital nasolacrimal duct obstruction with dacryoendoscope. Clin Ophthalmol. 2014;8:977-80.

12. Fujimoto M, Ogino K, Matsuyama H, Miyazaki C. Success rates of dacryoendoscopy-guided probing for recalcitrant congenital nasolacrimal duct obstruction. Jpn J Ophthalmol. 2016;60:274-9.

13. Ohtomo K, Ueta T, Nagahara M. Congenital nasolacrimal duct obstruction with fungal dacryoliths. Can J Ophthalmol. 2015;50: e7-8.

14. Matsumura N, Goto S, Yamane S, et al. High-resolution dacryoendoscopy for observation for pediatric lacrimal duct obstruction. Am J Ophthalmol Case Rep. 2016;1:23-5.

15. Heichel J, Bredehorn-Mayr T, Struck HG. Endoscopy of the lacrimal duct system in children. Klin Monbl Augenheilkd. 2015;232:881-5.

16. Cohen SW, Prescott R, Sherman M, Castillejos ME. Dacryoscopy. Ophthalmic Surg. 1979;10:57-63.

17. Fein W, Daykhovsky L, Papaioannou T, Beeder C, Grundfest WS. Endoscopy of the lacrimal outflow system. Arch Ophthalmol. 1992;110:1748-50.

18. Emmerich KH, Steinhauer J, Meyer-Rusenberg HW, Lüchtenberg M. Dacryoendoscopy-current status. Ophthalmologe. 1998;95:820-2.

19. Mimura M, Ueki M, Oku H, Sato B, Ikeda T. Indications for and effects of Nunchaku-style silicone tube intubation for primary acquired lacrimal drainage obstruction. Jpn J Ophthalmol. 2015;59:266-72.

20. MacEwen CJ, Young JDH, Barras CW, Ram B, White PS. Value of nasal endoscopy and probing in the diagnosis and management of children with congenital epiphora. $\mathrm{Br} \mathrm{J}$ Ophthalmol. $2001 ; 85: 314-8$.

21. Kashkouli MB, Beigi B, Parvaresh MM, et al. Late and very late initial probing for congenital nasolacrimal duct obstruction: what is the cause of failure? Br J Opthalmol. 2003;87:1151-3.

22. Kashkouli MB, Abtahi MB, Sianati H, et al. A novel one-stage obstruction-based endoscopic approach to congenital nasolacrimal duct obstruction. Ophthal Plast Reconstr Surg. 2017;33:350-4.

23. Alanon-Fernandez MA, Alanon-Fernandez FJ, Martinez-Fernan$\mathrm{dez}$, et al. Comparative study of primary intention lacrimal probing with and without nasal endoscopy. Acta Otorrinolaringol Esp. 2014;65:297-301.

24. Theodoropoulou S, Sutherland MS, Haddow K, Blaikie A. Success rates of endoscopic-assisted probing for congenital nasolacrimal duct obstruction in children. J Laryngol Otol. 2013;127:794-8.

25. Weiss AH, Baran F, Kelly J. Congenital nasolacrimal duct obstruction: delineation of anatomic abnormalities with 3dimensional reconstruction. Arch Ophthalmol. 2012;130:842-8.

26. Matsumura N, Goto S. Eiichi Uchio, Kyoko Nakajima, Takeshi Fujita, and Kazuaki Kadonosono. Cytokine profiles of tear fluid from patients with pediatric lacrimal duct obstruction. Invest Ophthalmol Vis Sci. 2017;58:252-6.

27. Fielding CA, Jones GW, McLoughlin RM, et al. Interleukin-6 signaling drives fibrosis in unresolved inflammation. Immunity. 2014;40:40-50.

28. Pediatric Eye Disease Investigator Group. A randomized trial comparing the cost-effectiveness of 2 approaches for treating unilateral nasolacrimal duct obstruction. Arch Ophthalmol. 2012;130:1525-33. 